\title{
Resultados de la implementación del reporte de sospecha de reacciones adversas a medicamentos por pacientes: revisión estructurada
}

\author{
Results of the implementation of the report of suspicion of \\ adverse drug reactions by patients: structured review
}

Daniel Pino-Marin ${ }^{1}$; Juliana Madrigal-Cadavid ${ }^{1}$; Mauricio Ceballos-R ${ }^{1}$; Alejandra Cano-P ${ }^{1}$; Pedro Amariles ${ }^{1}$

Forma de citar: Pino Marín D, Madrigal Cadavid J, Ceballos M, Cano A, Amariles P. Resultados de la implementación del reporte de sospecha de reacciones adversas a medicamentos por pacientes: revisión estructurada. Rev Univ Ind Santander Salud. 50(2): 136-142. doi: http://dx.doi.org/10.18273/revsal.v50n2-2018005 (c) (1)

\section{Resumen}

Objetivo: Identificar la contribución del reporte de reacciones adversas a medicamentos por pacientes, así como las barreras y facilitadores asociados con esta estrategia. Metodología: Revisión estructurada en PubMed/MedLine de artículos en inglés y/o español, hasta el 30/05/2017, con los términos: Pharmacovigilance reporting AND patient report. Resultados: Se obtuvieron 16 artículos con información relacionada con el objeto de estudio y se incluyeron tres artículos más considerados como relevantes. Se identificaron las principales barreras y facilitadores del paciente para reportar la sospecha de reacciones adversas a medicamentos, los efectos del reporte y las características del mismo. De igual forma, se describieron las diferencias y similitudes entre el reporte por profesionales de la salud y pacientes. Conclusiones: La implementación del informe por pacientes disminuye el sub-reporte en farmacovigilancia. Además, permite la participación activa y la integración de las experiencias del paciente con las reacciones adversas a los medicamentos.

Palabras clave: Farmacovigilancia; Efectos colaterales y reacciones adversas relacionados con medicamentos; Utilización de medicamentos; Atención ambulatoria; Participación del paciente.

\begin{abstract}
Objective: To identify the contribution of the report of suspected adverse drug reactions by patients, as well as the barriers and facilitators associated with this strategy. Methodology: Structured review based on PubMed / MedLine articles in English and Spanish, until 05/30/2017, using the keywords: Pharmacovigilance reporting AND patient report. Results: 16 articles were identified with information related to the object of study and three more were included taking into account their relevance. Main barriers and facilitators of patients for reporting suspected adverse drug reactions, the effects of the report and its characteristics, were identified. Besides, similarities and disparities between reports from health professionals and patients were described. Conclusions: The implementation of the

1. Universidad de Antioquia. Medellín, Colombia.

Correspondencia: Daniel Esteban Pino Marín. Dirección: Calle 67 n. 53-108, bloque 2-125. UDE Medellín. Teléfono: +57 3012505310 . Correo electrónico: daniel.pino@udea.edu.co.
\end{abstract}


report by patients decreases sub-reporting in pharmacovigilance. In addition, it allows the active participation and integration of patients' experiences about adverse drug reactions.

Keywords: Pharmacovigilance; Drug-related side effects and adverse reactions; Drug utilization; Ambulatory care; Patient participation.

\section{Introducción}

El logro de los objetivos terapéuticos con la utilización de los medicamentos requiere que sean efectivos y seguros. En este sentido, previo a su comercialización, se realizan estudios preclínicos y clínicos orientados a establecer su eficacia y seguridad. Sin embargo, posterior a su comercialización pueden aparecer efectos no identificados en dichos estudios; lo que se explica, entre otras, por las siguientes limitaciones: a) tiempo corto de exposición al medicamento, b) número pequeño de participantes, c) criterios de exclusión múltiples $\mathrm{y}, \mathrm{d})$ realización en condiciones diferentes a la práctica clínica habitual ${ }^{1,2}$. En este sentido, la farmacovigilancia (FV) se encarga de detectar, evaluar, entender y prevenir los efectos adversos y cualquier otro problema relacionado con los medicamentos una vez comercializados ${ }^{3}$.

El logro de los objetivos de la FV requiere de la recolección de información sobre el uso de los medicamentos y la aparición de sus efectos adversos. La notificación voluntaria, por parte de profesionales de la salud (PDS), es uno de los métodos orientados a cumplir con dicha finalidad. Esta estrategia, a pesar de presentar ventajas, tiene una importante limitación, el sub-reporte, el cual se estima en un $96 \%$ a nivel mundial ${ }^{4}$. En este contexto, las actividades educativas a PDS, la simplificación del formato de reporte, la retroalimentación al reportante se han utilizado como mecanismos para contrarrestar el sub-reporte 5 . Aunque, desde hace décadas algunos países han establecido la importancia del reporte de sospechas de reacciones adversa a medicamentos (RAM) por el paciente ${ }^{6}$, el tema ha tomado vigencia con la directiva de la Unión Europea 84/2010 ${ }^{7}$. En Colombia existe una propuesta de formato de reporte de sospechas RAM por pacientes ${ }^{8}$; sin embargo, similar a lo que sucede en la mayoría de países de la región, explícitamente no se tiene establecida la participación del paciente 7 .

De forma general, se acepta que el reporte de RAM por pacientes favorece el logro de los objetivos de la $\mathrm{FV}^{6}$; sin embargo, la información de los resultados, barreras y limitaciones asociadas a esta estrategia es fraccionada. Por ello, en países como Colombia, la sistematización de información relacionada con experiencias, de países en los que existe esta opción, podría favorecer la implementación del reporte de RAM por pacientes. En este contexto, el objetivo de esta revisión fue identificar la contribución del reporte de reacciones adversas a medicamentos por pacientes y las barreras y facilitadores asociadas de esta estrategia.

\section{Metodología}

\section{Tipo de estudio: Revisión estructurada}

Se realizó una revisión bibliográfica en PubMed/ MedLine de artículos publicados en inglés y/o español, hasta el 30/05/2017, con los siguientes términos de búsqueda en el titulo o resumen: Pharmacovigilance reporting AND patient report. Además, se incluyeron artículos consideradas como relevantes de las referencias bibliográficas de los artículos identificados.

\section{Criterios de inclusión}

Artículos relacionados con la contribución, barreras y facilitadores de la implementación del reporte de sospecha de RAM por pacientes.

\section{Criterios de exclusión}

Artículos relacionados con estrategias que excluyen al paciente como reportante, estrategias centradas en profesionales de la salud o con construcción del formato de reporte.

\section{Método de revisión}

Los artículos incluidos fueron evaluados de forma individual por cada investigador. Para ello, se revisaron los títulos y resúmenes de todos los artículos identificados para decidir su elegibilidad. Los artículos seleccionados se analizaron conjuntamente $\mathrm{y}$, por consenso entre todos los investigadores, se definió su inclusión. Para la recolección de la información se construyó una base de datos en Excel ® 2010, que contenía la siguiente información: a) autor/es, b) título, c) revista y año de publicación, d) país, e) contribución, f) barreras y facilitadores identificados y g) conclusiones relevantes. Posterior a esto se analizó la información y se agrupo por categorías. 


\section{Resultados}

La estrategia de búsqueda permitió identificar 84 artículos, de los cuales se accedió al texto completo de 83. De ellos, 16 aportaron información relacionada con el objeto de estudio, adicionalmente se incluyeron tres artículos más, considerados como relevantes. El flujo de la revisión se ilustra en la Figura 1.

\section{Contribuciones del reporte por pacientes en la farmacovigilancia}

Globalmente, existe evidencia del efecto positivo del reporte por parte del paciente en los programas de FV $^{11-15}$. Por ejemplo, en Holanda, entre 2004 y 2007 se generaron 2522 reportes, relacionados con 5401 $\mathrm{RAM}^{11}$; en el 2014, 4393 reportes fueron realizados por pacientes, equivalentes al $20 \%$ del total ${ }^{12,13}$; para el 2015 el número de reportes por pacientes aumentó aproximadamente a $8000^{12,14,15}$.

Por su parte, en Italia, se utilizó como estrategia al farmacéutico para promover el reporte de RAM por parte de pacientes. En esta dirección, el centro de FV de la región de Veneto recibió 2311 reportes de RAM realizados por pacientes $(46,7 \%$ del total de pacientes entrevistados por los farmacéuticos que habían referido una RAM), en comparación de los 226 enviados por otros PDS en el 2010 ${ }^{16}$; mientras que, entre el 2014 y 2015 se generaron 5127 reportes $^{12}$.

En Francia, durante la pandemia de influenza H1N1 en el 2009, se realizaron 4746 reportes al French Network of Pharmacovigilance Centres; de ellos, 1006 (21,2\%) fueron por pacientes ${ }^{17}$. Según datos de 2014-2015, los reportes por pacientes fueron $6082^{12}$.

En la Figura 1, se muestran los resultados de la contribución de esta estrategia en diferentes países pertenecientes al Uppsala Monitoring Centre durante el año 2014.

Igualmente, esta estrategia ha permitido establecer señales alertantes que contribuyen a mejorar el perfil de seguridad de los medicamentos ${ }^{15}$, entre estos están: a) enfermedad inflamatoria de vejiga asociado al uso de isotreonina, b) asociación de hiperparatiroidismo con el uso de litio, c) asociación entre ludopatía y el uso de pergolida, d) presencia de dolores de cabeza con el retiro de octreotido ${ }^{11}$, e) asociación entre agresión y el uso de vareniclina, f) asociación de alucinaciones visuales y uso de escopolamina, g) interacción medicamento-alimento, entre amlodipino y el jugo de pomelo ${ }^{7,18}$. Debido a esto, se han generado cambios en los insertos de los medicamentos, caso de la pergolida en Holanda ${ }^{11}$.

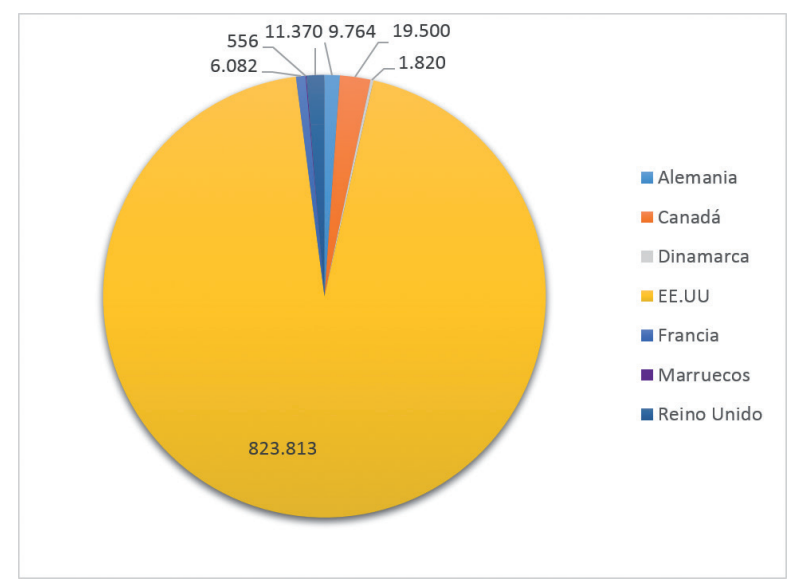

Figura 1. Contribución del reporte por pacientes en diferentes países durante $2014^{12,13}$.

\section{Barreras y facilitadores para el paciente en el reporte de RAM}

En general, como principales barreras relacionadas con el reporte por parte del paciente se ha identificado: a) escasa o pobre conciencia relacionada con un bajo conocimiento de los sistemas de reporte de RAM, b) desconocimiento de las agencias reguladoras y los formatos de reporte disponibles, c) poca o nula retroalimentación por parte de las agencias reguladoras, d) temor a causar algún tipo de desaprobación por parte de los profesionales de la salud, y e) costos de envío de reportes, especialmente en países en vía de desarrollo, ${ }^{9}$.

Igualmente, en estudios realizados con pacientes, con una media edad de 46 años (IC, o min-max), se han identificado que las principales limitaciones al momento de diligenciar un formato de reporte de RAM son: a) dificultad para recordar la fecha del evento adverso y el nombre del medicamento, la cual aumenta con el paso del tiempo de la ocurrencia del evento, b) extensión del formato de reporte y uso de términos complejos, c) falta de conocimiento en el manejo y diligenciamiento de formatos en línea ${ }^{10}$.

Por su parte, con respecto a los facilitadores, en Holanda se utilizó como estrategia para aumentar el reporte por pacientes, la transmisión de un mensaje relacionado con la experiencia de RAM graves, sufridas por pacientes al usar estatinas, lo que generó un aumento en el número de reportes realizados por pacientes, en unas 10 veces, aunque pasadas las semanas éste se normalizo ${ }^{21}$. 
Los principales motivos identificados para el reporte por parte del paciente, obedecen a: a) intenciones de participar activamente y contribuir a la disminución del sub-reporte, b) prevenir la aparición de las RAM en otros pacientes, especialmente las graves c) mejorar el perfil de seguridad de los medicamentos d) contribuir al mejoramiento de la práctica clínica de los PDS, e) obtener una retroalimentación sobre los reportes realizados, f) solicitud de los PDS, quienes fomentan a los pacientes a realizar sus reportes ${ }^{9,10,24}$. Igualmente, algunos autores establecen que: a) el 93,8\% de los pacientes opinó que, el reportar una RAM, puede prevenir que otros la presenten; b) el 97,9\% consideró que reportar contribuye a la generación de conocimiento; c) el $90,7 \%$ expresó que se sentían responsables de notificar; y d) el 92,5\% declaró que volverían a reportar una RAM en el futuro ${ }^{25,26}$.

Por su parte, Langen ${ }^{11}$, identificó que el reporte por pacientes facilita la expresión de síntomas difíciles de discutir con los PDS, especialmente los relacionados con la actividad sexual. De igual manera, establece que los pacientes refieren que su PDS no daba importancia a las RAM informadas, limitando su reporte ${ }^{10,11}$. En la Tabla 1, se muestran las principales barreras y facilitadores identificadas en la revisión.

Tabla 1. Barreras y facilitadores del reporte de RAM por pacientes ${ }^{9-11,22,24-26}$

\begin{tabular}{ll}
\hline \multicolumn{1}{c}{ Barreras } & \multicolumn{1}{c}{ Facilitadores } \\
\hline $\begin{array}{l}\text { Falta de conciencia } \\
\begin{array}{l}\text { Desconocimiento de las agencias y formatos de reporte } \\
\text { Temor }\end{array}\end{array}$ & $\begin{array}{l}\text { Uso de medios de comunicación masiva } \\
\text { Educación y estimulación con relación al reporte de RAM } \\
\text { Contribución al mejoramiento de la práctica clínica }\end{array}$ \\
$\begin{array}{l}\text { Falta de retroalimentación } \\
\text { Dificultad para recordar fechas y datos del medicamento }\end{array}$ & Contribución al uso adecuado de los medicamentos \\
$\begin{array}{l}\text { Formatos en lenguajes muy técnicos } \\
\text { Falta de formación en uso de tecnologías de la información y yeneración de conocimiento y prevención de aparición de } \\
\text { comunicación }\end{array}$ & \begin{tabular}{l} 
RAM en la comunidad \\
\hline
\end{tabular}
\end{tabular}

\section{Características de los reportes realizados por pacientes}

En general, los reportes realizados por pacientes se encuentran relacionados conmedicamentos ampliamente usados en el ámbito ambulatorio y de venta libre ${ }^{16}$; los grupos farmacológicos con mayor incidencia de reporte son estatinas, inhibidores selectivos de la recaptación de serotonina, beta bloqueadores, anticoagulantes e inhibidores de la bomba de protones ${ }^{11}$; además, algunos reportes son de agentes antineoplásicos o medios de contraste $^{16}$.

Con relación a la clasificación ATC (Anatomical, Therapeutic, Chemical classification system) de la OMS, los medicamentos con mayores reportes fueron: enfermedades del sistema nervioso (57,0\%), agentes psicotrópicos $(49,5 \%)$, antiinfecciosos $(7,5 \%)$, metabolismo y tracto digestivo (6,5\%), sistema respiratorio $(5,9 \%)$, dermatológico $(5,4 \%)$, cardiovascular $(4,8 \%)$, genitourinario y hormonas sexuales $(3,8 \%)$ y medicamentos del sistema musculo-esquelético $(3,2 \%)^{10}$. Por su parte, las cinco asociaciones más reportadas por los pacientes fueron: estatinas asociadas con mialgia y artralgia; paroxetina con aumento del peso, disminución de la libido y fatiga $^{11}$.
En un estudio realizado por Parretta, et al. encontraron que, de 18677 pacientes entrevistados, 2032 refirieron una RAM y de estos solo 716 realizaron el reporte. Estos reportes se encontraban relacionados con RAM esperadas (94.62\%) y no serias (91.28\%). Además, estaban relacionados con problemas gastrointestinales $(40,26 \%)$, afectaciones en piel y tejidos (31,54\%), alteraciones generales y del sistema nervioso (14,62 y 14,36\% respectivamente). Con relación a los medicamentos, los más frecuentes fueron amoxicilina/ ácido clavulánico, ketoprofeno, claritromicina, ácido acetilsalicílico, entre otros ${ }^{16,19}$. Finalmente, se ha identificado que los pacientes que viven en el área urbana, son más propensos a diligenciar de manera correcta y con información adecuada los formatos de reporte de $\mathrm{RAM}^{20}$.

\section{Diferencias y similitudes entre el reporte por profesionales de la salud y pacientes}

Langen, et al. identificaron que los pacientes generaron 2522 reportes, relacionados con 5401 RAM; en comparación con los 10635 reportes realizados por PDS, relacionados con 16722 RAM $^{11}$. En general, los reportes por pacientes contienen un nivel similar en la información clínica comparado con los realizados por los PDS ${ }^{11,14}$. 
La gravedad de las RAM no presentó diferencias significativas (19.5\% pacientes vs $21 \%$ PDS). Sin embargo, las diferencias encontradas estuvieron relacionadas con gravedad, debido a que los pacientes reportaron un mayor número de RAM que amenazaban la vida (5.2\% vs $2.7 \%)$, al igual que las causantes de discapacidad $(2.3 \%$ vs $0.4 \%)$. En cuanto a la recuperación del evento, los pacientes reportaron sin recuperación en mayor proporción que los PDS $(35.4 \% \text { vs } 16.7 \%)^{11,16,21}$. Igualmente se ha identificado que los pacientes centran la evaluación de las RAM en el impacto sobre la vida cotidiana, mientras que los PDS se centran más en evaluar la causalidad ${ }^{22}$.

Un trabajo buscó mediante un formato Web recolectar de manera anónima, las experiencias de los pacientes con relación al uso de antidepresivos. El formato contenía la información mínima necesaria para evaluar la temporalidad y causalidad de la RAM, además, contenía una escala likert de 5 puntos, para evaluar el impacto de la RAM en la calidad de vida del paciente. Al comparar los reportes realizados por los pacientes, con los realizados por los profesionales de la salud, durante el mismo periodo y sobre los mismos medicamentos, se identificó que los pacientes reportaron un mayor número de eventos como apatía, sudoración excesiva, inefectividad, somnolencia, insomnio, problemas sexuales y aumento del peso. Mientras que los PDS reportaron rash, prurito, anormalidades en pruebas de laboratorio, complicaciones musculares y articulares, desórdenes congénitos, problemas oculares, desórdenes extrapiramidales y menstruales ${ }^{23}$.

\section{Discusión}

La vinculación activa del paciente en el proceso de reporte de sospechas de RAM ha demostrado ser una alternativa que facilita el logro de los objetivos de la FV, no solo por el aumento en la cantidad de reportes, asociado a la disminución del sub-reporte, sino también, por la identificación de señales alertantes y de RAM que afectan su calidad de vida.

En los diferentes países donde se ha implementado este sistema, especialmente en Europa, los reportes por pacientes han evidenciado un incremento notorio en el número de reportes recibidos por los organismos de control. Por ejemplo, Holanda ha demostrado un compromiso notable y unos resultados extraordinarios. Durante el periodo de 2004/2007, recibió 2.522 y para el 2015, alcanzó a recibir 8000 reportes por pacientes ${ }^{12,14,15}$. Esto demuestra que el compromiso institucional y un adecuado sistema de información y educación al ciudadano, puede favorecer la inclusión del paciente en las políticas y estrategias en salud. Además de favorecer el incremento en el número de reportes, la perspectiva del paciente permite establecer señales alertantes que contribuyen a mejorar el perfil de seguridad de los medicamentos ${ }^{7,11,15,18}$, lo cual apunta específicamente al cumplimiento de los objetivos de la FV.

En este sentido, la identificación de las principales barreras relacionadas con el reporte de sospecha de RAM por parte de pacientes, podría favorecer la estructuración y ajuste de programas de información y educación al paciente con relación al uso de medicamentos, la aparición, manejo y reporte de RAM. Por tal razón, el objetivo de estos programas debe estar encaminado en la disminución de las barreras y en la potencialización de los facilitadores. En consecuencia, la voluntad del paciente de contribuir a la salud pública, de participar activamente y prevenir la aparición de las RAM en los demás usuarios de medicamentos ${ }^{18}$, son facilitadores que deben ser aprovechados por el sistema de salud, para permitir su participación y posible enriquecimiento de la práctica clínica con la perspectiva que podría aportar el paciente.

En Colombia, como ha sucedido en otros países, el reporte de RAM por pacientes podría aumentar la cantidad de reportes y la identificación de señales y alertas relacionadas con el uso de medicamentos, especialmente los de uso ambulatorio. En este aspecto, para el periodo de septiembre-octubre de 2016, el sistema nacional de farmacovigilancia recibió un total de 14.964 reportes, relacionados con 13.982 RAM de PDS. De éstos, 61\% fue realizado por Instituciones Prestadoras de Salud (IPS), 32\% por la industria farmacéutica, 4\% por las Empresa Social del Estado (ESE) y un 3\% por operadores logísticos ${ }^{27}$. Con relación a esto, Calderón, et al. recomiendan que es necesario mejorar la articulación de funciones entre todos los actores participantes en el uso de los medicamentos y hacer de la detección y el reporte de RAM una práctica sistemática, en especial de los medicamentos que han sido recientemente comercializados ${ }^{28}$. En el logro de esta finalidad el paciente podría favorecer y contribuir al mejoramiento del Programa Nacional de FV, debido a que es quien utiliza los medicamentos y experimenta las RAM, lo que genera información adicional, desde una perspectiva diferente que sirva como complemento a la información aportada por PDS. 


\section{Conclusión}

Globalmente se ha demostrado que la implementación del reporte por parte del paciente favorece la disminución del sub-reporte en FV y a identificar señales de alerta. Además, permite la participación activa del paciente y la integración de sus experiencias frente a las RAM, como complemento a la perspectiva de los PDS, específicamente, en el impacto que tienen estas en la calidad de vida, contribuyendo al perfil de seguridad de los medicamentos. De igual manera, la identificación de las barreras y facilitadores de esta estrategia, podría contribuir a la creación o ajuste de programas de información y educación al paciente con relación al uso de medicamentos y a la aparición, manejo y reporte de las RAM.

\section{Agradecimientos}

Fuente de apoyo financiero: El grupo Promoción y Prevención Farmacéutica recibió financiación del Comité para el Desarrollo de la Investigación (CODI) de la Universidad de Antioquia, convocatoria sostenibilidad (2014 a 2015).

\section{Conflictos de interés}

Los autores manifiestan que no existe ningún conflicto de intereses.

\section{Referencias}

1. Ivy SP, Siu LL, Garrett-Mayer E, Rubinstein L. Approaches to phase 1 clinical trial design focused on safety, efficiency, and selected patient populations: a report from the clinical trial design task of national cancer institute investigational drug steering committee. Clin Cancer Res. 2010;16(6): 1726-1736. doi: 10.1158/1078-0432.CCR-09-1961.

2. Vlahakes GJ. The value of phase IV clinical testing. N Engl J Med. 2006; 354(4): 413-415.

3. World Health Organization, the Uppsala Monitoring Centre. The importance of pharmacovigilance. Uppsala: World Health Organization, the Uppsala Monitoring Centre, 2002.

4. Hazell L, Shakir SA. Under-reporting of adverse drug reactions: a systematic review. Drug Saf. 2006; 29(5): 385-396.

5. Molokhia M, Tanna S, Bell D. Improving reporting of adverse drug reactions: systematic review. Clin Epidemiol. 2009; 1: 75-92

6. van Hunsel F, Härmark L, Pal S, Olsson S, van Grootheest K. Experiences with adverse drug reaction reporting by patients: an 11-country survey. Drug Saf. 2012; 35(1): 45-60.

7. Unión Europea. Official Journal of the European Union. Directive 2010/84/EU of the European Parliament and of the Council. 31 Dic 2010.

8. Pino-Marín D, León-Torres A, Amariles P. Propuesta de un formato para el reporte de sospecha de reacciones adversas a medicamentos por pacientes en Colombia. Rev Univ Ind Santander Salud. 2016; 48(3): 365-373. doi: http://dx.doi.org/10.18273/ revsal.v48n3-2016010.

9. Al Dweik R, Stacey D, Kohen D, Yaya S. Factors affecting patient reporting of adverse drug reactions: a systematic review. Br J Clin Pharmacol. 2017; 83(4): 875-883. doi: 10.1111/bcp.13159.

10. Yamamoto M, Kubota K, Okazaki M, Dobashi A, Hashiguchi M, Doi $\mathrm{H}$, et al. Patients views and experiences in online reporting adverse drug reactions: findings of a national pilot study in Japan. Patient Prefer Adherence. 2015; 9: 173-184. doi: 10.2147/PPA.S75761.

11. de Langen J, van Hunsel F, Passier A, de Jongvan den Berg L, van Grootheest K. Adverse drug reaction reporting by patients in the Netherlands: three years of experience. Drug Saf. 2008; 31(6): 515-524.

12. Banovac M, Candore G, Slattery J, Houÿez F, Haerry D, Genov G, et al. Patient Reporting in the EU: Analysis of EudraVigilance Data. Drug Saf. 2017; 40(7): 629-645. doi: 10.1007/s40264-0170534-1.

13. Matos C, Härmark L, van Hunsel F. Patient reporting of adverse drug reactions: an international survey of national competent authorities' views and needs. Drug Saf. 2016; 39(11): 1105-1116.

14. Rolfes L, van Hunsel F, van der Linden L, Taxis $\mathrm{K}$, van Puijenbroek E. The quality of clinical information in adverse drug reaction reports by patients and healthcare professionals: a retrospective comparative analysis. Drug Saf. 2017; 40(7): 607-614.

15. van Hunsel F, de Waal S, Härmark L. The contribution of direct patient reported ADRs to drug safety signals in the Netherlands from 2010 to 2015. Pharmacoepidemiol Drug Saf. 2017; 26(8): $977-$ 983. doi: $10.1002 /$ pds. 4236 .

16. Leone R, Moretti U, D’Incau P, Conforti A, Magro $\mathrm{L}$, Lora R, et al. Effect of pharmacist involvement on patient reporting of adverse drug reactions: first Italian study. Drug Saf. 2013; 36(4): 267-276. doi: 10.1007/s40264-013-0028-8.

17. Durrieu G, Palmaro A, Pourcel L, Caillet C, Faucher A, Jacquet A, et al. First French experience of ADR 
reporting by patients after a mass immunization campaign with Influenza $\mathrm{A}(\mathrm{H} 1 \mathrm{~N} 1)$ pandemic vaccines: a comparison of reports submitted by patients and healthcare professionals. Drug Saf. 2012; 35(10): 845-854. doi: 10.2165/11631800000000000-00000.

18. Cumber SL, Heffer SJ, Gandhi S, et al. The Yellow Card Scheme: experience of patient reporting of adverse drug reactions 5 years since launch [abstract]. Pharmacoepidemiol Drug Saf. 2010;19: S321.

19. Parretta E, Rafaniello C, Magro L, Coggiola Pittoni A, Sportiello L, Ferrajolo C, et al. Improvement of patient adverse drug reaction reporting through a community pharmacist-based intervention in the Campania region of Italy. Expert Opin Drug Saf. 2014;13 Suppl 1:S21-9.

20. Staniszewska A, Dąbrowska-Bender M, Olejniczak D, Duda-Zalewska A, Bujalska-Zadrożny M. Patient knowledge on reporting adverse drug reactions in Poland. Patient Prefer Adherence. 2016; 11: 47-53. doi: 10.2147/PPA.S117572.

21. van Hunsel F, Passier A, van Grootheest K. Comparing patients' and healthcare professionals' ADR reports after media attention: the broadcast of a Dutch television programme about the benefits and risks of statins as an example. Br J Clin Pharmacol. 2009; 67(5): 558-564. doi: 10.1111/j.13652125.2009.03400.x.

22. Rolfes L, Wilkes S, van Hunsel F, van Puijenbroek E, van Grootheest K. Important information regarding reporting of adverse drug reactions: a qualitative study. Int J Pharm Pract. 2014; 22(3): 231-233. doi: 10.1111/ijpp. 12056.

23. van Geffen ECG, van der Wal SW, van Hulten R, de Groot MCH, Egberts ACG, Heerdink ER. Evaluation of patients' experiences with antidepressants reported by means of a medicine reporting system. Eur J Clin Pharmacol. 2007; 63(12): 1193-1199. doi: 10.1007/s00228-007-0375-4.

24. Dweik RA, Yaya S, Stacey D, Kohen D. Spontaneous adverse drug reaction reporting by patients in Canada: a multi-method study-study protocol. Springerplus. 2016; 5: 213. doi: 10.1186/ s40064-016-1838-9.

25. van Hunsel F, van der Welle C, Passier A, van Puijenbroek E, van Grootheest K. Motives for reporting adverse drug reactions by patientreporters in the Netherlands. Eur J Clin Pharmacol. 2010; 66(11): 1143-1150. doi: 10.1007/s00228010-0865-7.

26. Mukherjee S, Sen S, Kalaiselvan V, Tripathi SK.
Consumer reporting of adverse drug reactions: a current perspective. IJGP. 2016; 10(3): 136-144.

27. Invima. Boletín de Farmaseguridad Vol 5 N5, 2016.

28. Calderón-Ospina C, Urbina-Bonilla A. La Farmacovigilancia en los últimos 10 años: actualización de conceptos y clasificaciones. Logros y retos para el futuro en Colombia. Rev Médica UIS. 2012; 24(1): 53-66. 